\title{
PHOX2B mutations and polyalanine expansions correlate with the severity of the respiratory phenotype and associated symptoms in both congenital and late onset Central Hypoventilation syndrome
}

\author{
I Matera, T Bachetti, F Puppo, M Di Duca, F Morandi, G M Casiraghi, M R Cilio, R Hennekam, \\ R Hofstra, J G Schöber, R Ravazzolo, G Ottonello, I Ceccherini
}

J Med Genet 2004;41:373-380. doi: 10.1136/jmg.2003.015412

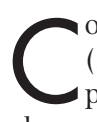
ongenital Central Hypoventilation syndrome (CCHS (MIM 209880)) is a rare disorder, with fewer than 200 patients currently reported worldwide, characterised by absence of adequate autonomic control of respiration with decreased sensitivity to hypercapnia and hypoxia, in the absence of neuromuscular or lung disease, or an identifiable brain stem lesion. ${ }^{1}$ Children with CCHS show an adequate ventilation while awake but hypoventilate during sleep. More severely affected children hypoventilate both when awake and during sleep. ${ }^{1}$ CCHS has been reported in association with several disorders, among which aganglionic megacolon (Hirschsprung disease, HSCR) and tumours of neural crest origin, reflecting a common molecular pathogenesis sustained by defects of one or more genes that control the correct development of neural crest derived cell lineages. ${ }^{1-3}$

A genetic aetiology has long been hypothesised for CCHS based on recurrence reported in siblings, in half siblings and in affected children born to women with CCHS. ${ }^{2-6}$ More recently, a generalised autonomic nervous system (ANS) imbalance has been observed among children with CCHS and an increased incidence of ANS dysfunctions (ANSD) reported among relatives of 56 patients with CCHS, as against relatives of 56 matched controls. ${ }^{7}$ A family transmission study has shown that the risk of developing an ANSD symptom including CCHS, regarded as the most severe expression of ANS imbalance, mainly depends on the genotype at a major locus, while significant residual variants could be due to additional minor genes, modifying loci effects or environmental factors. ${ }^{8}$

Genes involved in the ANS development, like the RET proto-oncogene, its ligand GDNF, the Endothelin 3 gene, the Brain Derived Neurotrophic Factor $(B D N F)$ and the $R N X$ genes, have been tested and a few mutations found, showing no cosegregation with the disease phenotype in CCHS families. ${ }^{9-13}$

The $P H O X 2 B$ gene encodes a 314 amino acids paired box homeodomain transcription factor which is expressed in the developing hindbrain and peripheral nervous system as well as in all noradrenergic centres and visceral motor and branchiomotor neurones of the cranial nerves. Its expression has also been detected in neuronal groups, such as those involved in the medullary control reflexes of autonomic functions. ${ }^{14}$ Phox $2 b-/-$ mice have shown lack of intestinal innervation and of all central and peripheral neurones that express noradrenergic traits. ${ }^{15}$ Two heterozygous frameshift mutations and 16 polyalanine triplet duplications of 5-9 residues, occurred in a 20 alanine tract in the $C$ terminus of the $P H O X 2 B$ gene, have been described in a total of 29 patients with CCHS (62\%). ${ }^{16}$ More recently, a mutation screening of the $P H O X 2 B$ gene performed in 10 patients has

\section{Key points}

- We have carried out mutation screening of the PHOX2B gene in a set of 27 patients affected with congenital Central Hypoventilation syndrome (CCHS), three of whom showed association with Hirschsprung's disease (HSCR), and also including three familial cases with affected sibling pairs and three late onset central hypoventilation cases (LOCHS).

- Three heterozygous frameshift mutations, two of which occurred de novo, and 22 in-frame changes leading to 5-13 polyalanine expansions of the $P H O X 2 B$ gene in 25 out of 27 CCHS patients studied (93\%) have been identified. Polyalanine triplet expansion has also been detected in the affected siblings of two familial cases and in two asymptomatic parents, one of whom is the mother of a sporadic case.

- A genotype-phenotype correlation is shown between the size of the PHOX2B expanded allele and the severity of both the respiratory phenotype and associated symptoms, and the age of onset.

- We demonstrate that in individuals heterozygous for length variants of the polyalanine stretch of PHOX2B exon 3, the largest allele is always prone to unsuccessful amplification by DNA polymerase. An improved methodological approach is proposed to avoid misleading in vitro amplification that can cause mistakes in recording individuals' genotypes.

- $P H O X 2 B$ is confirmed as the major gene in CCHS pathogenesis. Autosomal dominant inheritance with reduced penetrance is demonstrated.

revealed polyalanine expansions in four patients with CCHS and one frameshift mutation in a fifth patient $(50 \%),{ }^{17}$ while 65 polyalanine expansions and one nonsense mutation of the same gene have been detected on a total of 67 patients with CCHS $(98.5 \%) .^{18}$ Thus, $P H O X 2 B$ is confirmed as playing a crucial role in the correct development of the central respiratory control system and appears to be a master regulatory gene of the ANS.

Abbreviations: ANS, autonomic nervous system; ANSD, ANS dysfunctions; CCHS, congenital Central Hypoventilation syndrome; HSCR, Hirschsprung's disease; LOCHS, late onset Central Hypoventilation syndrome; SNP, single nucleotide polymorphism 
Here, we report a mutation screening of the coding region of the $P H O X 2 B$ gene in a total of 27 unrelated patients affected with CCHS, including three familial cases with affected sibling pairs and three cases with late onset CHS (LOCHS). ${ }^{19}$ Results support the notion of a simple Mendelian inheritance in CCHS with dominant, incompletely penetrant PHOX $2 B$ molecular defects recurring in the vast majority of the patients and their families. Moreover, the size of the polyalanine expansions detected correlates with the degree of phenotypic severity.

\section{METHODS}

\section{Subjects}

We have analysed 27 individuals from Italy, Germany and the Netherlands affected with CCHS, isolated or in association with several autonomic nervous system dysfunctions. Twenty one of them have isolated CCHS, three presented with CCHS and HSCR, while the other three had a late onset of the disease. Three isolated CCHS cases are familial, showing affected siblings.

The diagnostic criteria of the American Thoracic Society statement on $\mathrm{CCHS}^{1}$ were used and the diagnosis of CCHS was confirmed for each case by the referring clinician. Sixty unrelated individuals who were phenotypically normal were recruited from the blood donors of the Istituto Gaslini and used as controls. DNA was extracted from peripheral blood lymphocytes according to standard procedures.

Our study protocol was approved by the ethics committee of the Department of Internal Medicine of the University of Genova and informed consent was obtained from all patients' parents before study enrolment.

\section{Mutational screening and molecular analysis}

To amplify the three coding regions, including intron-exon boundaries, we designed four PCR primer pairs (table 1) on the basis of the PHOX2B sequence available in GenBank (accession number NT_022782) and using the Primer Express 2.0 software program.

Standard PCR reactions were set up in a total volume of $50 \mu \mathrm{l}$ containing $200 \mathrm{ng}$ of genomic DNA, $1 \mu \mathrm{M}$ of primers, $1 \times$ buffer, $200 \mu \mathrm{M}$ of each dNTP except for $\mathrm{G}$, which was present at $150 \mu \mathrm{M}+50 \mu \mathrm{M}$ of 7-deaza-dGTP (Roche), and $1.25 \mathrm{U}$ of Taq polymerase (PE Biosystems), and run for 35 cycles at $95{ }^{\circ} \mathrm{C}(45 \mathrm{~s})$, the annealing temperature reported in table 1 ( $1 \mathrm{~min})$, and $72{ }^{\circ} \mathrm{C}(1 \mathrm{~min})$.

PCR reactions specific for GC rich templates were set up in a total volume of $50 \mu \mathrm{l}$ containing $200 \mathrm{ng}$ of genomic DNA, $400 \mathrm{nM}$ primers, $1 \times$ of GC-RICH PCR buffer and $1 \mathrm{M}$ GCRICH resolution (GC-RICH PCR System, Roche), $200 \mu \mathrm{M}$ of each dNTP and $2 \mathrm{U}$ of GC-RICH PCR enzyme mix (Roche), and run for 30 cycles at $95{ }^{\circ} \mathrm{C}(30 \mathrm{~s}), 60{ }^{\circ} \mathrm{C}(30 \mathrm{~s})$, and $72{ }^{\circ} \mathrm{C}$ (1 min).

PCR fragments were screened for mutations by direct sequencing, using the Big Dye Terminator cycle sequencing kit (Applied Biosystems) on an ABI 3100 DNA automated Sequencer. PCR products were column purified before sequencing (Microcon-PCR, Millipore).

The fifth primer pair reported in table $1(145 \mathrm{~F}+279 \mathrm{R})$ was used to amplify a small portion of exon 3. $15 \mu \mathrm{l}$ of this $120 \mathrm{bp}$ PCR product were run on $8 \%(\mathrm{w} / \mathrm{w})$ polyacrylamide gel, and DNA bands on the gel were developed using ethidium bromide.

We cloned the exon 3 PCR product (primers 10F+3.3R) using DNA from patient 23 as a template, into the TOPO TA vector (Invitrogen) following the manufacturer's instructions. Plasmid DNA isolated from 35 clones was sequenced as described above.

A restriction cleavage with AvaII of the 627 bp exon 3 PCR amplimer (primers $10 \mathrm{~F}+3.3 \mathrm{R}$ ) of all patients, was carried out and analysed through $4 \%$ agarose gel electrophoresis.

\section{RESULTS}

Mutation analysis of PHOX2B gene in CCHS patients A mutation screening of the three exons spanning the entire coding sequence of the $P H O X 2 B$ gene has been performed in 27 CCHS patients, including three LOCHS cases, by direct DNA sequencing analysis. We found three nucleotide changes lying in the exon 3 and leading to out of frame translation (fig 1A).

One heterozygous $\mathrm{C}$ deletion and one heterozygous $\mathrm{G}$ insertion have been detected in two CCHS patients (27 and 21) within intervals c.614-618 and c.862-866 of the coding sequence, respectively (fig $1 \mathrm{~B}$ ). In the former case, the frame shift creates a premature stop codon and results in a 307 amino acid truncated protein, with the last 101 amino acids not belonging to the PHOX2B sequence. In the latter case, the resulting frame shift relocates the stop codon 132 bp beyond its natural end, leading to disruption of $8.3 \%$ of the protein Cterminus. Analysis of the two patients' parents has revealed that the c.614-618delC was inherited from the unaffected mother, while the c.862-866insG occurred de novo in the proband (data not shown). A 38 bp heterozygous deletion within exon 3, removing nucleotides $721-758$ of the coding sequence and predicted to destroy the reading frame, has been found in the third patient (24) (fig 1B). His parents were found to carry no mutant $P H O X 2 B$ allele, following agarose gel analysis (fig $1 C$ ) and direct sequencing (data not shown) of the exon 3 amplification product.

\section{Expansion of the alanine stretch in our CCHS patients} To verify the presence of a possible expansion of the polyalanine tract in the exon 3 of a proportion of the 24 remaining non-mutant patients with CCHS, a 120 bp PCR product, resulting from amplification with primers $145 \mathrm{~F}$ and 279R (table 1), was run on $8 \%$ acrylamide gel (fig 2A). Patients 8, 23, and 28 did show an extra band (with heteroduplex formation) corresponding to a size compatible with an expanded band. Indeed, DNA sequence of exon 3 had

Table 1 Primers and PCR conditions

\begin{tabular}{llll}
\hline \multicolumn{2}{l}{ Primers: name and sequence $\left(\mathbf{5}^{\prime} \rightarrow \mathbf{3}^{\prime}\right)$} & \\
\hline Amplimers & Size, bp & Forward & Reverse \\
\hline Exon $\mathbf{1}^{*}$ & 320 & 35F: GGCTGAGCCATCCAGAACCT & 35R: CCTATATACGGGCGGAAAGGC \\
Exon 2* & 260 & PH2F: TGCCGGCTGATTGCTCAC & PH2R: AGCGGGGTCGGTTCCAGG \\
Exon 3† & 627 & 10F: TGCTCACCGTCTCTCCTCC & 3.3R: TACCCGCTCGCCCACTCG \\
Exon 3† & 458 & 22F: GCACTGACCCGGACAGCACT & 3.3R: TACCCGCTCGCCCACTCG \\
Exon 3* $^{*}$ & 120 & 145F: AACCCGGCAAGGGCGGC & 60 \\
\end{tabular}

* Standard PCR reactions

†GC rich templates $P C R$ reactions

$\mathrm{T}_{\mathrm{a}}$, temperature of annealing 
A

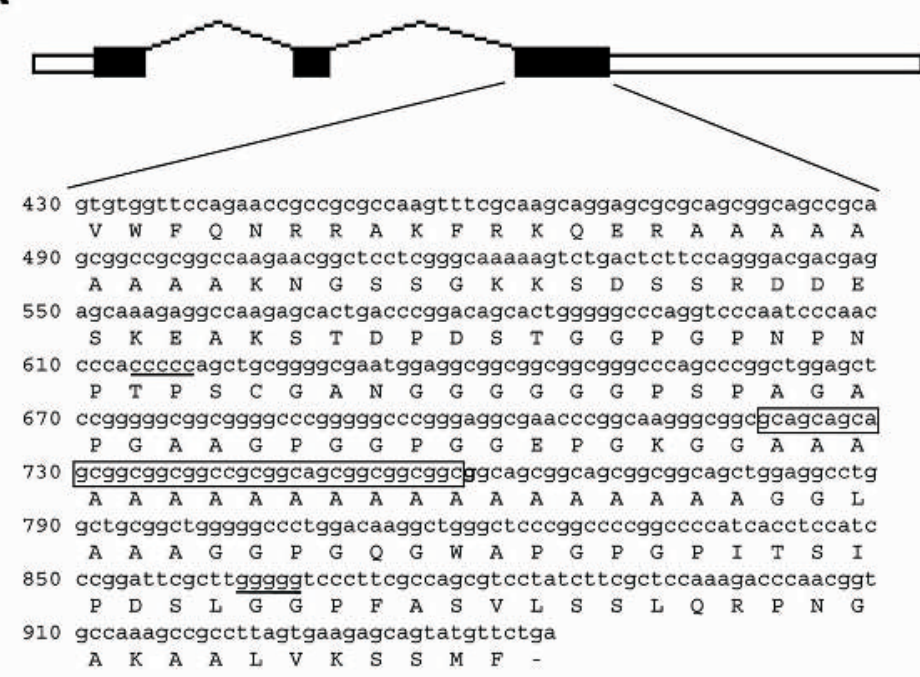

C

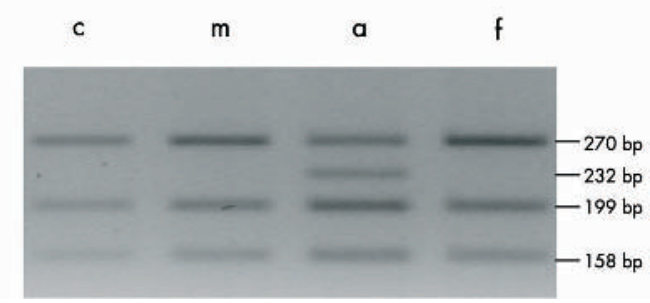

B
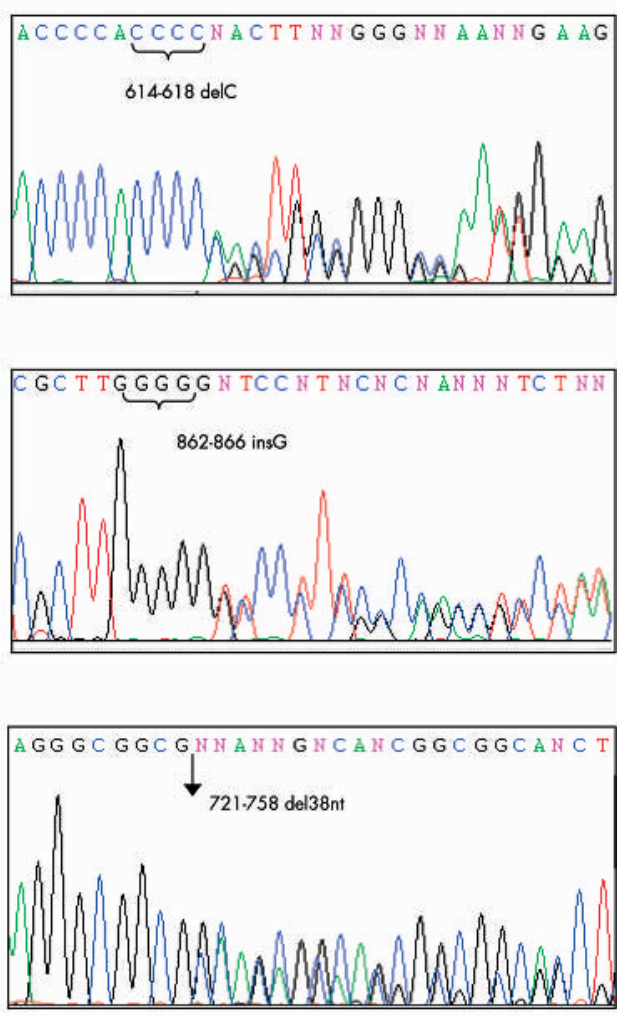

Figure 1 PHOX2B frameshift mutations detected in the exon 3 of three CCHS patients. (A) Nucleotide and amino acid sequences of the coding portion of the third and last exon of the $P H O X 2 B$ gene are shown, along with the position of the single nucleotide deletion and insertion detected (underlined) and the extent of the 38 bp interstitial deletion (boxed). (B) Sequence chromatograms of partial portions of exon 3 of the PHOX2B gene. From top to bottom: c.614-618delC, c.862-866insG, and c.721-758del38nt. The position of the mutations is indicated. (C) Avall enzymatic digestion of the $627 \mathrm{bp}$ DNA fragment encompassing the c.721-758del38nt was obtained from genomic DNA of both a control (c) and the mother $(\mathrm{m})$ and the father (f) of the affected child (a). Unlike the first three samples, showing only bands derived from normal alleles (158 bp, $199 \mathrm{bp}$, and $270 \mathrm{bp}$ ), enzymatic digestion of the patient's sample showed an additional $232 \mathrm{bp}$ band, corresponding to the deleted allele, as predicted from the sequence analysis.

shown, in a proportion of CCHS DNAs including 2, 3, 7, 11, 16,23 , and 28 , but not 8 , very weak and variable evidences of the expansion of a number of alanines, ranging from 5 to 6 residues, with respect to the wild-type sequence. Such a putative expansion had the shape of a double shifted sequence displaying an intensity, in terms of height of the single peaks, varying from $10 \%$ to $30 \%$ of the main wild-type sequence, as shown in fig $2 \mathrm{~B}$, and could be observed, after either polyacrylamide gel electrophoresis or sequencing experiments, in seven individuals out of 27 , and shown to recur from $25 \%$ to $100 \%$ of the times the DNA from a same individual was resequenced.

\section{Cloning of an "alanine-expanded" allele}

To confirm that the expanded sequence we observed did correspond to a germline expansion of the PHOX2B allele, carried by patients in their constitutive genomic DNA, we undertook the cloning of an exon 3 PCR product (primers 10F+3.3R) using DNA from patient 23, which had already shown evidence of alanine expansion, as a template. We used a single nucleotide polymorphism (SNP) identified by us during the mutational screening, a c $870 \mathrm{C}>\mathrm{A}$ change leading to a silent substitution in codon P290 of exon 3, to distinguish between the two alleles derived from DNA of patient 23, who had proved to be heterozygous for this variant $(\mathrm{C} / \mathrm{A})$. After amplification, we cloned the PCR product and sequenced 35 clones, each corresponding to one of the two single alleles originally present in the genomic DNA. Two combinations of polyalanine length and SNP alleles were obtained: the wild-type allele, containing 20 normal alanine residues, associated with the A variant in 28 clones, while the expanded allele, containing 25 alanine residues, associated with the $\mathrm{C}$ variant in the other seven clones. This has allowed us to verify that expansion was always associated with the same allele, as expected in case of a genomic expansion and to confirm a ratio of 1:4 of expanded:wild-type alleles which reflects the fact that longer (expanded) fragments are underrepresented in the PCR product. Moreover, the absence of different combinations of SNP alleles and polyalanine tracts allows the exclusion of both mosaicism in patient 23 and a possible artefact originated in vitro during DNA amplification

\section{Amplification of the exon 3 GC-rich region}

The presence of a polyalanine expansion seems to result in the lack of amplification of the mutant allele. The sequence of the $P H O X 2 B$ exon 3 , and especially of its polyalanine tract, is highly GC rich (fig lA). A probable explanation for the weak or absent amplification observed during the sequencing of our DNA samples is, therefore, that the presence of strong secondary structures might inhibit the action of the DNA polymerase during DNA amplification. In the attempt to overcome this problem we had already modified the standard amplification reaction by adding $10 \%$ glycerol or 7 -DeazadGTP or increasing the annealing temperature, or both, but this might have not been sufficient to remove the impeding secondary structures resulting in false negative responses. So, to maximise the chance of correct amplification of both 
A
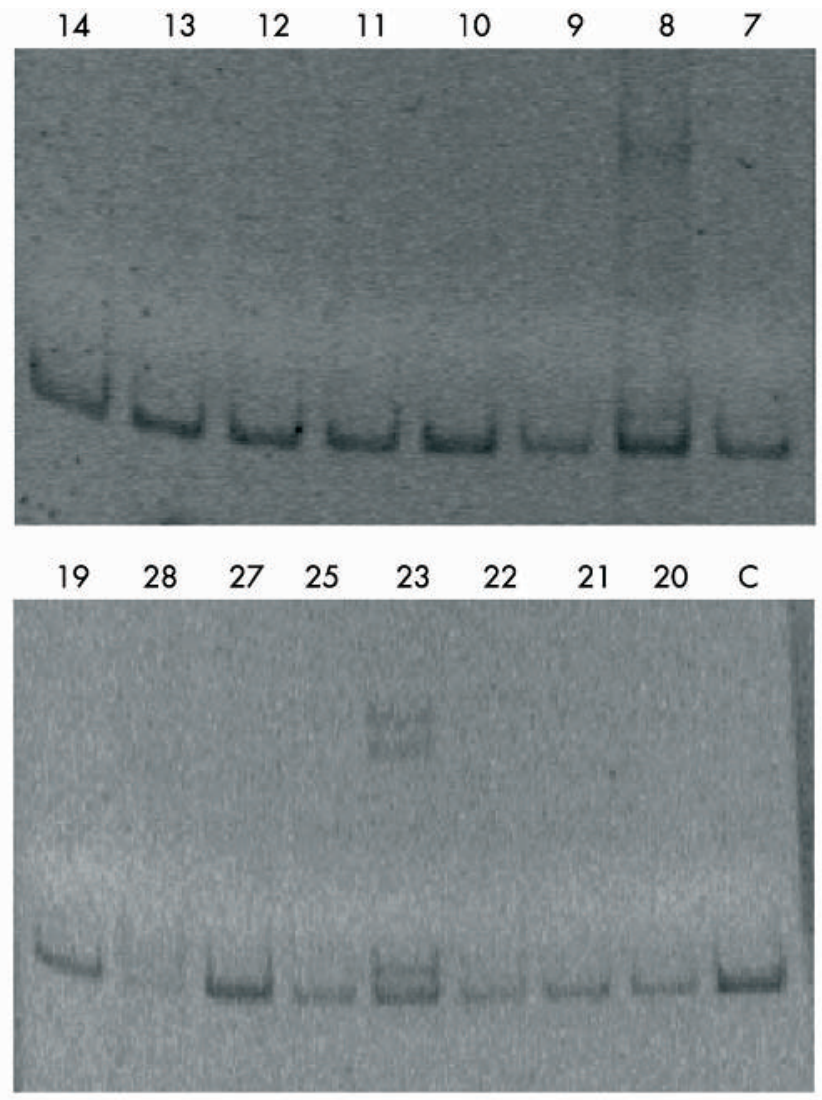

B

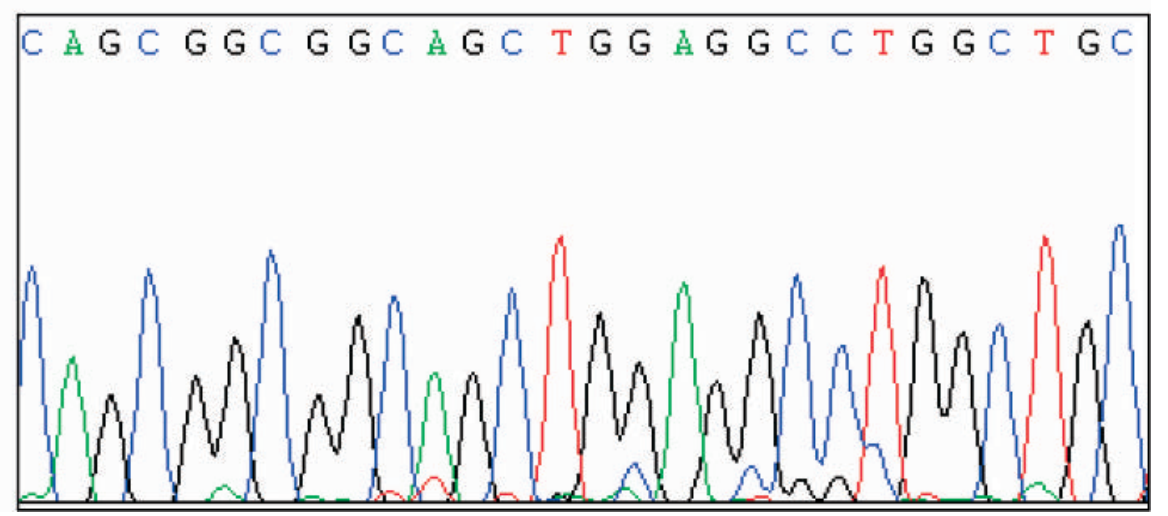

Figure 2 Expansion of the 20 alanine residues tract of PHOX2B. (A) Polyacrylamide Gel Electrophoresis of a 120 bp fragment obtained with primers $145 \mathrm{~F}$ and $279 \mathrm{R}$ in DNA from 16 patients and 1 control. Patient 23 , and to a lesser extent also patients 8 and 28 , show extra large bands accompanied by heteroduplexes at a higher size. Such a pattern is compatible with the presence of two PCR products and presumably with an alanine expansion. (B) 28 nucleotides sequence chromatogram of an exon 3 portion of the PHOX2B gene (selected at the end of the polyalanine stretch) obtained from patient 23 , who shows a double band in the polyacrilamide gel electrophoresis analysis of the $145 \mathrm{~F}+279 \mathrm{R} P C R$ product (see panel (A)). The sequence pattern of patient 23 is in agreement with an expanded PCR product present with an intensity much lower than the wild-type sequence.

alleles, we set up a PCR reaction making use of a couple of primers $(10 \mathrm{~F}+3.3 \mathrm{R})$ delimiting the whole exon 3 and of a system properly developed for GC rich templates. Direct sequencing of DNA amplification products thus obtained allowed us to detect polyalanine triplet expansions in samples which had previously shown neither variants nor weak double sequences. In particular, we identified 22 in frame changes leading to polyalanine expansions resulting from duplications, inserted in different positions of the polyalanine tract, ranging from 5 to 13 alanine residues and involving the last 15-39 bp before the insertion. An AvaII enzymatic digestion of the $627 \mathrm{bp}$ exon 3 PCR amplimer allowed us to confirm presence of extra bands as shown in fig 3A. In particular, bands of $158 \mathrm{bp}, 199 \mathrm{bp}$, and $270 \mathrm{bp}$, this latter containing the 20 alanine stretch, were obtained from enzymatic digestion of wild-type amplification products while expanded alleles could be recognised by the replacement of the largest fragment with an extra band of size variable from 285 to $309 \mathrm{bp}$ (duplicates from 5 to 13 alanine residues). A summary of all the polyalanine expansions observed in our patients is reported in figure 3B. Polyalanine triplet expansion has also been detected in the affected siblings of two familial CCHS cases (4F and $11 \mathrm{~F}$ ). Inheritance by the asymptomatic mother could be proven for one of 
A

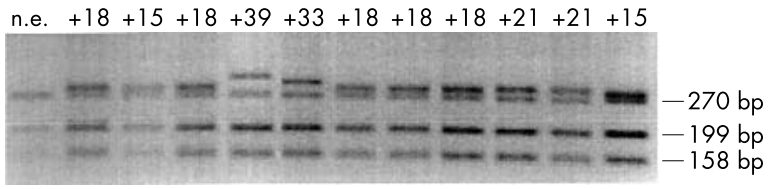

B

A $=$ GCA $\mathrm{G}=\mathrm{GCG} \quad \mathrm{C}=\mathrm{GCC}$ T $=\mathrm{GCT}$ Ala codons

AAAGGGCGAGGGGAGAGGAT 20 polyala tract

\begin{tabular}{|c|c|c|}
\hline & dup nt & Patient number \\
\hline AAAGGGCGAGGGGA GAGGAGAGGAT & +15 & $1,4 F, 15,19,23$ \\
\hline AAAGGGCGAGGGGAGGGGAGAGGAT & $+15^{\star}$ & $11 F, 16$ \\
\hline AAAGGGCAAGGGCGAGGGGAGAGGAT & +18 & $2,7,28$ \\
\hline AAAGGGCGAGGGCGAGGGGAGAGGAT & $+18^{\star}$ & $3,8,9,12,18$ \\
\hline AAAGGGCGAGGGGAGAGGGGAGAGGAT & +21 & $5,6,20,22,13$ \\
\hline AAAGGGCGAGGGGAGGGCGAGGGGAGAGGAT & +33 & 10 \\
\hline AAAGGGCGAGGGGAGAGGGCGAGGGGAGA & +39 & 14 \\
\hline
\end{tabular}

Figure 3 Expansion of the 20 alanine residues tract of $P H O X 2 B$ in CCHS patients. (A) Examples of various duplication lengths (+15 to +39 nucleotides corresponding to +5 to +13 alanine residues) are provided and shown as extra bands of size variable from 285 to $309 \mathrm{bp}$,

obtained after enzymatic digestion with Avall of the 627 bp PCR product of $\mathrm{PHOX} 2 \mathrm{~B}$ exon 3 (primers $10 \mathrm{~F}+3.3 \mathrm{R}$ ) and migration on a $4 \%$ agarose gel (158 bp, $199 \mathrm{bp}$, and $270 \mathrm{bp}$ are the expected, wild-type bands). On the top of each lane the length of duplication is reported as number of nucleotides. (B) Summary of the polyalanine expansions observed in our patients. The duplicated codons are shown in grey boxes. An asterisk is used to distinguish between different positions at which expansions of the same size have occurred.

these, while, in the other case, the mother does not carry the Ala expansion and DNA from the father is not available (data not shown). The asymptomatic mother of a sporadic case with an 18 bp expansion of the alanine stretch has resulted in carrying the same mutant allele (data not shown). Patients 17 (LOCHS) and 25 (CCHS) showed neither mutations nor expansions of the polyalanine stretch in exon 3. Loss of heterozygosity could be excluded at a SNP locus identified by us in exon 3 (c.552C > T leading to S184S) only for patient 25, who was found to be heterozygous $(\mathrm{C} / \mathrm{T})$. A summary of all the results is reported in table 2 , along with clinical details of each patient.

None of the mutations found in the present study were present in 120 normal control chromosomes. On the other hand, in agreement with a mechanism of non-homologous recombination generating the polyalanine expansion in disease associated mutant alleles, and according to what had already been reported, ${ }^{16-18}$ an alanine triplet contraction was found in one control DNA.

\section{Selective amplification of the shortest allele in heterozygous individuals}

During our mutational screening we had already collected evidence to support the fact that in individuals heterozygous for length variants of the polyalanine region of the $P H O X 2 \mathrm{~B}$ exon 3, the largest allele might have undergone unsuccessful amplification by DNA polymerase during the PCR reaction.

To confirm such hypothesis, we have performed PCR amplification of three DNA samples, selected to be heterozygous for length variants of the polyalanine stretch, using two different methods: the standard protocol and the GC rich system protocol. As shown in fig 4, by using the standard protocol we could observe no amplification from the wildtype allele of a control's DNA bearing a contraction of seven alanine residues (control c93), the expanded allele of a patient's DNA with a duplication of five alanine residues (patient 3), or the wild-type allele of the patient DNA with the 38 bp deletion (patient 24). Therefore, under standard conditions of amplification, each of the three individuals was found to be homozygous for the shortest of his or her two alleles. In fact, using the GC rich system protocol, two different alleles could be amplified from each of the same DNA samples, thus confirming the heterozygous status of the three individuals at the polyalanine tract locus (fig 4).

\section{DISCUSSION}

Congenital Central Hypoventilation syndrome (CCHS) is a rare disorder characterised by abnormal ventilatory response to hypoxia and hypercapnia, often associated with other autonomic nervous system dysfunctions. ${ }^{1}$

A mutational screening of the PHOX2B gene in a set of 29 CCHS cases has revealed heterozygous 5-9 alanine expansions within a stretch of 20 alanine in the $C$ terminus of the gene in 16 patients, and heterozygous frameshift mutations in two patients $(62 \%) .{ }^{16}$ More recently, two similar studies have reported both polyalanine expansions and a few missense, nonsense and out-of-frame nucleotide changes in exon 3 of the PHOX2B gene, reflecting mutation frequencies of $50 \%$ and $98.5 \%$ in 10 and 67 patients respectively. ${ }^{17} 18$

Here, we report the identification of three heterozygous frameshift mutations and 22 in frame changes leading to polyalanine expansions of the $\mathrm{PHOX} 2 \mathrm{~B}$ gene in a total of 27 CCHS patients studied, including three LOCHS cases (93\%). Two of the three frameshift mutations, a c.862-866insG and a 38 bp deletion of nucleotides c.721-758, have occurred de novo in two probands, while the third one, a novel c.614618delC, was inherited from the asymptomatic mother. Of the remaining 24 patients, 20 have 5-7 extra alanine residues in the polyalanine stretch of $P H O X 2 B$ exon 3, in addition to two other patients showing 11 and 13 polyalanine expansions. We have not detected expansions of fewer than five residues. Polyalanine triplet expansions have also been detected in the affected siblings of two familial CCHS cases. Inheritance from an asymptomatic parent could be proven for only one of these cases. Parents of sporadic cases have been found to carry no $P H O X 2 B$ molecular changes with the exception of the asymptomatic mothers of two patients with CCHS, one already mentioned with the c.614-618delC, and the other with an expansion of 18 alanine residues, who demonstrated transmission of the mutant allele to their affected children.

These observations provide a straightforward confirmation of simple dominant inheritance in CCHS with incomplete penetrance. So, the actual large proportion of sporadic cases might be explained by the very high mortality of patients with CCHS at a very young age or during the perinatal period until a few decades ago.

Two cases, one familial CCHS and one LOCHS, have been found to carry no $\mathrm{PHOX} 2 \mathrm{~B}$ molecular changes but we cannot exclude yet that defects either impairing correct expression or splicing of the gene, or residing outside the transcription unit (position effects) are present. Genetic heterogeneity could also explain the non-mutant patients.

As expected on the basis of data already reported, ${ }^{7}$ in addition to the respiratory phenotype each mutant patient shows a different and variable range of symptoms, mostly regarded as ANS dysfunctions (table 2). It is noticeable that the patient carrying the $38 \mathrm{bp}$ deletion (24) is the most seriously affected, with complete apnoea during sleep, severe hypoventilation during wakefulness and an extremely severe form of HSCR with total aganglionosis from duodenum to anus. Also patients 10 and 14, carrying the 11 th and 13th largest polyalanine expansions respectively, show an extremely severe respiratory phenotype with the need of 
Table 2 Clinical and molecular characterisation of our CCHS patients

\begin{tabular}{|c|c|c|c|c|c|c|}
\hline $\begin{array}{l}\text { Country of } \\
\text { origin }\end{array}$ & Patients & Ventilation & ANSD & Gastro-intestinal symptoms & Mutation & Transmission \\
\hline 1 & 01 LOCHS & during sleep & & & dup15 & de novo \\
\hline i & 02 & during sleep & 0 & & dupl8 & de novo \\
\hline 1 & 03 & during sleep & $\begin{array}{l}\text { dysrhythmia, loss of } \\
\text { consciousness }\end{array}$ & & dup18* & de novo \\
\hline 1 & $04 \mathrm{~F}$ & during sleep & O & constipation & dup15 & inherited \\
\hline 1 & 05 & during sleep & $\begin{array}{l}\text { O, decreased heart rate } \\
\text { variability }\end{array}$ & & dup21 & de novo \\
\hline I & 06 & during sleep & 0 & & dup21 & de novo \\
\hline$i$ & 07 & during sleep & & & dupl 18 & de novo \\
\hline i & 08 & during sleep & & & dupl $8^{*}$ & de novo \\
\hline I & 09 & during sleep & & & dupl 1* & inherited \\
\hline i & $10^{\dagger}$ & $24 \mathrm{hr}$ & O & constipation & dup33 & de novo \\
\hline G & $11 \mathrm{~F}$ & during sleep & & & dup15* & $?$ \\
\hline G & 12 & during sleep & & & dupl $8^{*}$ & ? \\
\hline G & 13 & $24 \mathrm{hr}$ & & & dup21 & $?$ \\
\hline G & 14 & $24 \mathrm{hr}$ & & HSCR & dup39 & $?$ \\
\hline 1 & 15 LOCHS & during sleep & & & dupl5 & de novo \\
\hline i & & during sleep & loss of consciousness & abdominal pain & dupl5* & de novo \\
\hline i & 17 LOCHS & during sleep & & & ne & \\
\hline i & 18 & during sleep & & & dup18* & de novo \\
\hline I & 19 & during sleep & & gastroesophageal reflux & dupl5 & de novo \\
\hline i & 20 & during sleep & $\begin{array}{l}\text { O, loss of consciousness, } \\
\text { altered sweating }\end{array}$ & dysphagia & dup21 & $?$ \\
\hline I & 21 & during sleep & $\begin{array}{l}\text { O, dysrhythmia, loss of } \\
\text { consciousness }\end{array}$ & constipation & ins $G$ & de novo \\
\hline G & 22 & during sleep & & HSCR & dup21 & de novo \\
\hline I & 23 & during sleep & altered perception of pain & constipation & dupl5 & de novo \\
\hline i & $24^{\ddagger}$ & $24 \mathrm{hr}$ & O & HSCR & del38 & de novo \\
\hline $\mathrm{NL}$ & $25 \mathrm{~F}$ & during sleep & & & ne & \\
\hline $\mathrm{NL}$ & $27^{\S}$ & during sleep & & & delC & inherited \\
\hline i & 28 & during sleep & O & & dupl8 & de novo \\
\hline
\end{tabular}

I, Italy; G, Germany; NL, The Netherlands; F, familial case; LO-CHS, late onset $\mathrm{CHS}$; O, ocular anomalies

${ }^{*}$ distinguishes expansions of similar size but different localisation within the polyalanine stretch ${ }^{\dagger}$ Growth hormone deficiency; ${ }^{\ddagger}$ died at 4 months; ${ }^{\S}$ tetralogy of Fallot; ne, not expanded

ventilatory support during the day time, the latter patient presenting HSCR too. In the attempt to draw a genotypephenotype correlation, we subdivided, on the basis of available information, patients into two groups according to

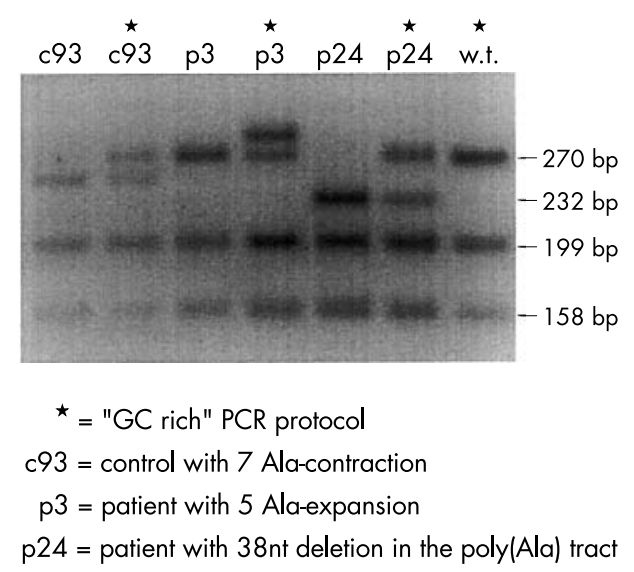

Figure 4 Non-random amplification of the shortest allele in heterozygous individuals. Wild-type and extra bands, obtained on a $4 \%$ agarose gel electrophoresis after enzymatic digestion with Avall of a 627 bp fragment (primers 1OF and 3.3R) are shown. Three DNA samples have been amplified using two different PCR methods: the standard protocol and the GC rich system protocol. Using the standard protocol, we could not observe any amplification from the wild-type allele in a control DNA bearing a contraction of seven alanine residues (c93), the expanded allele in a patient DNA with an expansion of five alanine residues (3), or the wild-type allele in the patient DNA with the 38 bp deletion (24). In contrast, using the GC rich system protocol, two different alleles could be amplified from each DNA sample thus confirming the heterozygous status of the three individuals at the polyalanine tract locus. their capacity to maintain sufficient oxygenation and ventilation for at least 30-60 minutes of sleep after removing mechanical ventilator support. ${ }^{20}$ In particular, in the most severely affected group, in which hypoxia and hypercapnia occurred after a few minutes of spontaneous ventilation, there are children affected with Hirschsprung disease and children with awake hypoventilation. This is the case of the patient carrying the 38 bp deletion, the two patients with the largest polyalanine expansions already mentioned and of some patients showing 6 or 7 alanine duplications. On the other hand, patients included in the least severely affected group, with children able to sustain 30-60 minutes of spontaneous ventilation before the $\mathrm{PCO}_{2}$ rises to $65 \mathrm{mmHg}$ in non-rapid eye movement sleep, neither presented with Hirschsprung nor demonstrated inadequate ventilation while awake, and all of them showed polyalanine expansions of $15 \mathrm{bp}$ (five alanine residues). This is also the case of the two LOCHS patients found to carry five extra Ala residues, who showed the first mild respiratory symptoms when two years old or later. Therefore, the differential diagnosis between LOCHS $^{19}$ and CCHS needs to be revisited since, at least at the respiratory level, the two so far clinically distinct conditions seem to reflect the same molecular disorder. Overall, these observations confirm the hypothesis of a genotype-phenotype correlation with phenotypic severity increasing with the polyalanine expansion size, and are in agreement with similar observations, recently made on the basis of the mean number of ANSD symptoms and the daily duration of ventilatory support. ${ }^{18}$

The 20 trinucleotide repeat of the polyalanine tract in PHOX $2 B$ is imperfect (fig 1 ). Therefore, replication slippage is unlikely and expansions are most probably caused by recombination between two mispaired alleles (unequal crossing over), as already proposed..$^{21} 22$ Such a mispairing mechanism is expected to result in expansions as well as 
contractions, a phenomenon that has been reported so far only in healthy individuals, and observed also in our control set, in terms of the presence of a rare polymorphism having no effect on the phenotype.

The existence of a mutational hotspot in the region coding for the polyalanine domain of $P H O X 2 B$ needs to be postulated since $92 \%$ of all mutations we detected so far in this gene leads to variations in the length of the polyalanine tract. There are at least nine genes in which alanine expansions have been shown to cause human disease. ${ }^{22}$ Moreover, 31 out of 98 sequences coding for polyalanine domains in the human genome have been found polymorphic, thus suggesting the opportunity of screening similar sequences in different pathologies. ${ }^{22}$ Although polyalanine tracts are common in transcription factors, neither their normal function nor the effects of their expansion are understood. Polyalanine tracts have been found in repression motifs of several proteins containing homeodomains and demonstrated, in some cases, to be responsible for the repression of target genes. ${ }^{23}$ In other cases, polyalanine tracts act as flexible spacer elements between functional domains. ${ }^{24}$ Thus, polyalanine expansions observed in $P H O X 2 B$ may either directly interfere with protein-protein interactions, distort an essential secondary structure, or act as dominant negative factors. The correlation between phenotypic severity and expansion size observed in CCHS suggests that tracts of 25 or more polyalanines might confer a progressive gain of function to $P H O X 2 B$, a hypothesis which will require further studies.

During our study we have collected proof that polyalanine expansion corresponds to a $P H O X 2 B$ allele carried by patients in their constitutive genomic DNA. Indeed, at first in our sequencing experiments alanine expansions had the appearance of an unclear background lying behind the normal DNA sequence in a small proportion of patients. Under the hypothesis that in individuals heterozygous for defects involving length changes of the polyalanine stretch of exon 3, the largest allele is always prone to unsuccessful amplification by DNA polymerase, and to avoid incorrect interpretation of the genotype of some patients and controls, we have developed a proper PCR protocol. Results reported in fig 4 have confirmed our hypothesis, showing different amplification outcomes when using different experimental protocols: when DNA amplification occurs under standard procedures, the shortest product is always observed while the largest amplimer can remain undetectable, as in the case of alanine expanded against wild-type alanine stretches and of wild-type alanine stretches against both the del38 allele and an alanine contracted tract in a control (fig 4). Such a misleading methodological aspect can cause mistakes in recording individuals' genotypes and we therefore suggest great care when approaching $P H O X 2 B$ mutational screening and especially for $P H O X 2 B$ molecular diagnosis in relatives of affected children. Indeed, following observation of recurrence of $P H O X 2 B$ defects and potential transmission of the CCHS phenotype to progeny, an accurate detection of $P H O X 2 B$ mutations represents the ground for genetic counselling, which has become indispensable for couples with an affected child to determine the recurrence risk to a fetus.

In conclusion, $P H O X 2 B$ is confirmed as the major gene in CCHS pathogenesis and autosomal dominant inheritance with reduced penetrance is now demonstrated. Moreover, a correlation between phenotypic severity and expansion length of the polyalanine tracts can be assumed and used to predict onset and progression of the disease in mutation carriers. Finally, molecular testing and genetic counselling has now become feasible, thus giving hopes to many families with CCHS.

\section{ACKNOWLEDGEMENTS}

We are extremely grateful to all the families who have participated in this study and the clinicians who have reported details of patients and provided blood samples. We want to thank Francesca Schena and Giuseppe Santamaria for their technical help.

\section{Authors' affiliations}

I Matera, T Bachetti, F Puppo, M Di Duca, R Ravazzolo, I Ceccherini, Laboratorio di Genetica Molecolare, Istituto G Gaslini, Genova, Italy M Di Duca, Laboratorio di Fisiopatologia dell'Uremia, Istituto G Gaslini, Genova Italy

F Morandi, UO Pediatria, Ospedale "Sacra Famiglia", Erba (CO), Italy G M Casiraghi, UO Anestesia e Rianimazione, Ospedale Mandic, Merate (Lecco), Italy

M R Cilio, Divisione di Neurologia, Ospedale Bambino Gesu', Roma, Italy

R Hennekam, Departments of Paediatrics and Clinical Genetics, University of Amsterdam, The Netherlands

R Hofstra, Department of Medical Genetics, University of Groningen, The Netherlands

J G Schöber, Kinderklinik Dritter Orden (vormals Lachnerklinik),

München, Germany

R Ravazzolo, Dipartimento di Pediatria e CEBR, Università di Genova, Italy

G Ottonello, UO Anestesia e Rianimazione, Istituto G Gaslini, Genova, Italy

This study was begun with the financial support of Telethon Italy (grant no E791) which is gratefully acknowledged. The completion of our work has been guaranteed by a grant obtained from Compagnia di San Paolo. Finally, we gratefully acknowledge the financial support of the European Community (contract no QLG1-CT-2001-01646) and of the Ministry of University (FIRB project to RR).

Conflicts of interest: none declared.

Correspondence to: Isabella Ceccherini, Ph.D, Laboratorio Genetica Molecolare, Istituto G Gaslini, L.go G Gaslini, 5, 16148 Genova, Italy; isa.c@unige.it

Revised version received 14 November 2003

Accepted for publication 17 November 2003

\section{REFERENCES}

1 Weese-Mayer DE, Shannon DC, Keens TG, Silvestri JM. Idiopathic congenital Central Hypoventilation syndrome. Am J Respir Crit Care Med 1999;160:368-73.

2 Haddad GG, Mazza NM, Defendini R, Blanc WA, Driscoll JM, Epstein MA, Epstein RA, Mellins RB. Congenital failure of automatic control of ventilation, gastrointestinal motility and heart Rate. Medicine (Baltimore) 1978;57:517-26.

3 Bower RJ, Adkins JC. Ondine's curse and neurocristopathy. Clin Pediatr (Phila) 1980;19:665-8.

4 Hamilton J, Bodurtha JN. Congenital central hypoventilation syndrome and Hirschsprung's disease in half sibs. J Med Genet 1989;26:272-4.

5 Weese-Mayer DE, Silvestri JM, Marazita ML, Hoo JJ. Congenital central hypoventilation syndrome: inheritance and relation to sudden infant death syndrome. Am J Med Genet 1993:47:360-77.

6 Silvestri JM, Chen ML, Weese-Mayer DE, McQuitty JM, Carveth HJ, Nielson DW, Borowitz D, Cerny F. Idiopathic congenital central hypoventilation syndrome: the next generation. Am J Med Genet 2002;112:46-50

7 Weese-Mayer DE, Silvestri JM, Huffman AD, Smok-Pearsall SM, Kowal MH Maher BS, Cooper ME, Marazita ML. Case/control family study of autonomic nervous system dysfunction in idiopathic congenital central hypoventilation syndrome. Am J Med Genet 2001;100:237-45.

8 Marazita ML, Maher BS, Cooper ME, Silvestri JM, Huffman AD, SmokPearsall SM, Kowal MH, Weese-Mayer DE. Genetic segregation analysis of autonomic nervous system dysfunction in families of probands with idiopathic congenital central hypoventilation syndrome. Am J Med Genet 2001;100:229-36

9 Bolk S, Angrist M, Xie J, Yanagisawa M, Silvestri JM, Weese-Mayer DE Chakravarti A. Endothelin-3 frameshift mutation in congenital central hypoventilation syndrome. Nat Genet 1996;13:395-6.

10 Amiel J, Salomon R, Attie T, Pelet A, Trang H, Mokhtari M, Gaultier C, Munnich A, Lyonnet S. Mutations of the RET-GDNF signalling pathway in Ondine's curse. Am J Hum Genet 1998;62:715-7.

11 Sakai T, Wakizaka A, Matsuda H, Nirasawa Y, Itoh Y. Point mutation in exon 12 of the receptor tyrosine kinase proto-oncogene RET in OndineHirschsprung syndrome. Pediatrics 1998;101:924-6.

12 Weese-Mayer DE, Bolk S, Silvestri JM, Chakravarti A. Idiopathic congenital central hypoventilation syndrome: evaluation of brain-derived neurotrophic 
factor genomic DNA sequence variation. Am J Med Genet 2002; 107:306-10

13 Matera I, Bachetti T, Cinti R, Lerone M, Gagliardi L, Morandi F, Motta M, Mosca F, Ottonello G, Piumelli R, Schober JG, Ravazzolo R, Ceccherini I. Mutational analysis of the RNX gene in congenital central hypoventilation syndrome. Am J Med Genet 2002;1 13:178-82.

14 Brunet JF, Pattyn A. Phox2 genes from patterning to connectivity. Curr Opin Genet Dev 2002; 12:435-40

15 Pattyn A, Morin X, Cremer H, Goridis C, Brunet JF. The homeobox gene Phox $2 \mathrm{~b}$ is essential for the development of autonomic neural crest derivatives. Nature 1999;399:366-70.

16 Amiel J, Laudier B, Attie-Bitach T, Trang H, de Pontual L, Gener B, Trochet D, Etchevers H, Ray P, Simonneau M, Vekemans M, Munnich A, Gaultier C, Lyonnet $\mathrm{S}$. Polyalanine expansion and frameshift mutations of the paired-like homeobox gene PHOX2B in congenital central hypoventilation syndrome. Nat Genet 2003;33:459-61

17 Sasaki A, Kanai M, Kijima K, Akaba K, Hashimoto M, Hasegawa H, Otaki S, Koizumi T, Kusuda S, Ogawa Y, Tuchiya K, Yamamoto W, Nakamura T, Hayasaka K. Molecular analysis of congenital central hypoventilation syndrome. Hum Genet 2003;114:22-6.

18 Weese-Mayer DE, Berry-Kravis EM, Zhou L, Maher BS, Silvestri JM, Curran ME, Marazita ML. Idiopathic congenital central hypoventilation syndrome: analysis of genes pertinent to early autonomic nervous system embryologic development and identification of mutations in PHOX2b. Am J Med Genet 2003;123A:267-78.

19 Katz ES, McGrath S, Marcus CL. Late-onset central hypoventilation with hypothalamic dysfunction: a distinct clinical syndrome. Pediatr Pulmonol 2000;29:62-8.

20 Gaultier C, Trang-Pham H, Praud JP, Gallego J. Cardiorespiratory control during sleep in the congenital Central Hypoventilation syndrome. Proceedings from the first international symposium on the congenital Central Hypoventilation syndrome-New Orleans, LA, May 11, 1996. Pediat Hypoventilation syndrome
Pulmonol 1997;23:140-2

21 Warren ST. Polyalanine expansion in synpolydactyly might result from unequal crossing-over of HOXD13. Science 1997; 275:408-9.

22 Lavoie H, Debeane F, Trinh QD, Turcotte JF, Corbeil-Girard LP, Dicaire MJ, Saint-Denis A, Page M, Rouleau GA, Brais B. Polymorphism, shared functions and convergent evolution of genes with sequences coding for polyalanine domains. Hum Mol Genet 2003;12:2967-79.

23 Han K, Manley JL. Functional domains of the Drosophila Engrailed protein EMBO J 1993;12:2723-33.

24 Karlin S, Burge C. Trinucleotide repeats and long homopeptides in genes and proteins associated with nervous system disease and development. Proc Natl Acad Sci U S A 1996:93:1560-5. 